\title{
First joint search for gravitational-wave bursts in LIGO and GEO 600 data
}

\author{
B Abbott ${ }^{1}, \mathbf{R}$ Abbott ${ }^{1}$, R Adhikari $^{1}$, P Ajith $^{2}$, B Allen $^{2,3}$, G Allen $^{4}$, \\ R Amin ${ }^{5}$, S B Anderson', W G Anderson ${ }^{3}$, M A Arain ${ }^{6}, M_{\text {Araya }}{ }^{1}$, \\ H Armandula ${ }^{1}$, P Armor ${ }^{3}$, Y Aso ${ }^{7}$, S Aston $^{8}$, P Aufmuth ${ }^{9}$, C Aulbert ${ }^{2}$, \\ S Babak ${ }^{10}$, S Ballmer ${ }^{1}$, H Bantilan ${ }^{11}$, B C Barish ${ }^{1}$, C Barker $^{12}$, D Barker ${ }^{12}$, \\ B Barr $^{13}$, P Barriga ${ }^{14}$, M A Barton ${ }^{13}$, I Bartos ${ }^{7}$, M Bastarrika $^{13}$, \\ K Bayer $^{15}$, J Betzwieser ${ }^{1}$, P T Beyersdor ${ }^{16}$, I A Bilenko ${ }^{17}$, G Billingsley ${ }^{1}$, \\ R Biswas $^{3}$, E Black ${ }^{1}$, K Blackburn $^{1}$, L Blackburn ${ }^{15}$, D Blair ${ }^{14}$, B Bland ${ }^{12}$, \\ T P Bodiya ${ }^{15}$, L Bogue ${ }^{18}$, R Bork ${ }^{1}, \mathbf{V}$ Boschi $^{1}, \mathbf{S}$ Bose $^{19}$, P R Brady ${ }^{3}$, \\ V B Braginsky ${ }^{17}, \mathrm{~J} \mathrm{E} \mathrm{Brau}^{20}, \mathrm{M}_{\text {Brinkmann }}{ }^{2}$, A Brooks $^{1}$, D A Brown $^{21}$, \\ G Brunet $^{15}$, A Bullington ${ }^{4}$, A Buonanno ${ }^{22}$, O Burmeister ${ }^{2}$, R L Byer ${ }^{4}$, \\ L Cadonati ${ }^{23}$, G Cagnoli ${ }^{13}$, J B Camp ${ }^{24}, \mathbf{J ~ C a n n i z z o}^{24}, \mathbf{K}_{\text {Cannon }}{ }^{1}$, \\ J Cao $^{15}$, L Cardenas ${ }^{1}$, T Casebolt ${ }^{4}$, G Castaldi ${ }^{25}$, C Cepeda ${ }^{1}$, E Chalkley ${ }^{13}$, \\ P Charlton ${ }^{26}, \mathbf{S}$ Chatterji ${ }^{1}, \mathbf{S}$ Chelkowski ${ }^{8}, \mathbf{Y}$ Chen $^{10,27}, \mathbf{N ~ C h r i s t e n s e n ~}^{11}$, \\ D Clark ${ }^{4}$, J Clark $^{13}$, T Cokelaer $^{28}$, R Conte $^{29}$, D Cook $^{12}$, T Corbitt $^{15}$, \\ D Coyne $^{1}$, J D E Creighton ${ }^{3}$, A Cumming ${ }^{13}$, L Cunningham ${ }^{13}$, \\ R M Cutler ${ }^{8}$, J Dalrymple $^{21}$, K Danzmann ${ }^{2,9}$, G Davies $^{28}$, D DeBra $^{4}$, \\ J Degallaix ${ }^{10}, \mathbf{M}_{\text {Degree }}{ }^{4}, \mathbf{V}$ Dergachev $^{30}, \mathbf{S}$ Desai $^{31}, \mathbf{R}$ DeSalvo ${ }^{1}$, \\ S Dhurandhar ${ }^{32}$, M Díaz ${ }^{33}$, J Dickson ${ }^{34}$, A Di Credico ${ }^{21}$, A Dietz ${ }^{28}$, \\ F Donovan ${ }^{15}$, K L Dooley ${ }^{6}$, E E Doomes ${ }^{35}$, R W P Drever ${ }^{36}$, I Duke ${ }^{15}$, \\ J-C Dumas ${ }^{14}$, R J Dupuis ${ }^{1}$, J G Dwyer ${ }^{7}$, C Echols ${ }^{1}$, A Effler ${ }^{12}$, P Ehrens ${ }^{1}$, \\ E Espinoza $^{1}$, T Etzel ${ }^{1}$, T Evans ${ }^{18}$, S Fairhurst ${ }^{28}$, Y Fan $^{14}$, D Fazi $^{1}$, \\ H Fehrmann ${ }^{2}$, M M Fejer ${ }^{4}$, L S Finn ${ }^{31}$, K Flasch ${ }^{3}$, N Fotopoulos ${ }^{3}$,

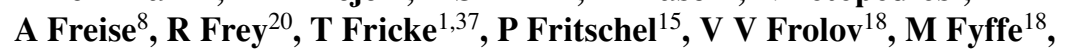 \\ J Garofoli ${ }^{12}$, I Gholami ${ }^{10}$, J A Giaime ${ }^{5,18}$, S Giampanis ${ }^{37}$, K D Giardina ${ }^{18}$, \\ K Goda $^{15}$, E Goetz ${ }^{30}$, L Goggin 1 , G González ${ }^{5}$, S Gossler ${ }^{2}$, R Gouaty ${ }^{5}$, \\ A Grant ${ }^{13}$, S Gras ${ }^{14}$, C Gray ${ }^{12}$, M Gray $^{34}$, R J S Greenhalgh ${ }^{38}$, \\ A M Gretarsson ${ }^{39}$, F Grimaldi $^{15}, \mathbf{R}_{\text {Grosso }}{ }^{33}, \mathbf{H}_{\text {Grote }^{2}}, \mathbf{S}_{\text {Grunewald }}{ }^{10}$, \\ M Guenther ${ }^{12}$, E K Gustafson ${ }^{1}$, R Gustafson ${ }^{30}$, B Hage ${ }^{9}$, J M Hallam $^{8}$,

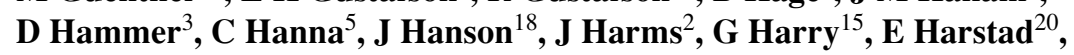 \\ K Hayama $^{33}$, T Hayler ${ }^{38}$, J Heefner ${ }^{1}$, I S Heng ${ }^{13}$, M Hennessy ${ }^{4}$,

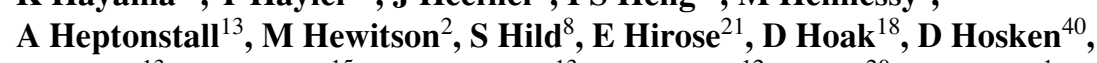

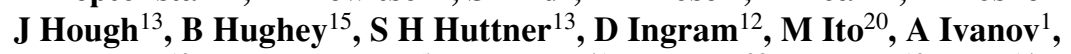 \\ B Johnson ${ }^{12}$, W W Johnson ${ }^{5}$, D I Jones ${ }^{41}$, G Jones ${ }^{28}$, R Jones ${ }^{13}$, L Ju ${ }^{14}$, \\ P Kalmus $^{7}$, V Kalogera ${ }^{42}$, S Kamat ${ }^{7}$, J Kanner ${ }^{22}$, D Kasprzyk ${ }^{8}$, \\ E Katsavounidis ${ }^{15}$, K Kawabe ${ }^{12}$, S Kawamura ${ }^{43}$, F Kawazoe $^{43}$, W Kells ${ }^{1}$,

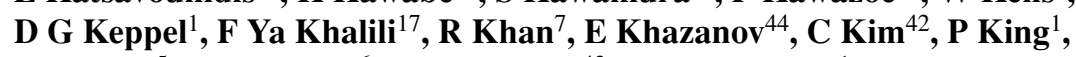 \\ J S Kissel ${ }^{5}$, S Klimenko ${ }^{6}$, K Kokeyama ${ }^{43}$, V Kondrashov ${ }^{1}$, \\ R K Kopparapu ${ }^{31}$, D Kozak ${ }^{1}$, I Kozhevatov ${ }^{44}$, B Krishnan ${ }^{10}$, P Kwee 9 , \\ P K Lam ${ }^{34}$, M Landry ${ }^{12}$, M M Lang ${ }^{31}$, B Lantz ${ }^{4}$, A Lazzarini ${ }^{1}$, M Lei ${ }^{1}$,
}


N Leindecker ${ }^{4}$, V Leonhardt ${ }^{43}$, I Leonor $^{20}, \mathrm{~K}_{\text {Libbrecht }}{ }^{1}, \mathbf{H}$ Lin $^{6}$, P Lindquist $^{1}$, N A Lockerbie ${ }^{45}$, D Lodhia ${ }^{8}$, M Lormand ${ }^{18}, \mathbf{P}$ Lu $^{4}$, M Lubinski ${ }^{12}$, A Lucianetti ${ }^{6}$, H Lück ${ }^{2,9}$, B Machenschalk ${ }^{2}$, M MacInnis ${ }^{15}$, M Mageswaran ${ }^{1}$, K Mailand ${ }^{1}$, V Mandic ${ }^{46}$, S Márka ${ }^{7}$, Z Márka ${ }^{7}$, A Markosyan ${ }^{4}$, J Markowitz ${ }^{15}$, E Maros ${ }^{1}$, I Martin ${ }^{13}, \mathbf{R}$ M Martin 6 , J N Marx ${ }^{1}$, K Mason ${ }^{15}$, F Matichard ${ }^{5}$, L Matone ${ }^{7}$, R Matzner ${ }^{47}$, N Mavalvala ${ }^{15}$, R McCarthy ${ }^{12}$, D E McClelland ${ }^{34}$, S C McGuire ${ }^{35}$, M McHugh $^{48}$, G McIntyre ${ }^{1}$, G McIvor ${ }^{47}$, D McKechan ${ }^{28}$, K McKenzie ${ }^{34}$, T Meier ${ }^{9}$, A Melissinos ${ }^{37}$, G Mendell ${ }^{12}$, R A Mercer ${ }^{6}$, S Meshkov $^{1}$, C J Messenger ${ }^{2}$, D Meyers ${ }^{1}$, J Miller ${ }^{1,13}$, J Minelli ${ }^{31}$, S Mitra ${ }^{32}$, V P Mitrofanov ${ }^{17}$, G Mitselmakher ${ }^{6}$, R Mittleman $^{15}$, O Miyakawa ${ }^{1}$, B Moe $^{3}$, S Mohanty ${ }^{33}$, G Moreno ${ }^{12}$, K Mossavi ${ }^{2}$, C MowLowry ${ }^{34}$, G Mueller $^{6}$, S Mukherjee ${ }^{33}$, H Mukhopadhyay ${ }^{32}$, H Müller-Ebhardt ${ }^{2}$,

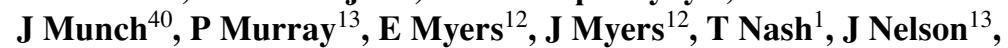
G Newton $^{13}$, A Nishizawa ${ }^{43}$, K Numata ${ }^{24}$, J O'Dell ${ }^{38}$, G Ogin ${ }^{1}$, B O'Reilly ${ }^{18}$, R O'Shaughnessy ${ }^{31}$, D J Ottaway ${ }^{15}$, R S Ottens ${ }^{6}$, H Overmier ${ }^{18}$, B J Owen ${ }^{31}$, Y Pan ${ }^{22}$, C Pankow ${ }^{6}$, M A Papa ${ }^{3,10}$,


T Petrie ${ }^{31}$, I M Pinto ${ }^{25}$, M Pitkin ${ }^{13}$, H J Pletsch ${ }^{2}$, M V Plissi ${ }^{13}$, F Postiglione ${ }^{29}$, M Principe ${ }^{25}$, R Prix ${ }^{2}$, V Quetschke ${ }^{6}$, F Raab ${ }^{12}$, D S Rabeling ${ }^{34}$, H Radkins ${ }^{12}$, N Rainer ${ }^{2}$, M Rakhmanov ${ }^{50}$,

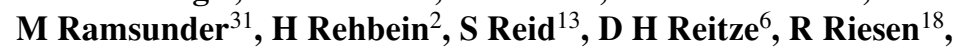
K Riles ${ }^{30}$, B Rivera ${ }^{12}$, N A Robertson ${ }^{1,13}$, C Robinson ${ }^{28}$, E L Robinson ${ }^{8}$, S Roddy ${ }^{18}$, A Rodriguez ${ }^{5}$, A M Rogan ${ }^{19}$, J Rollins ${ }^{7}$, J D Romano ${ }^{33}$, J Romie ${ }^{18}$, R Route ${ }^{4}$, S Rowan ${ }^{13}$, A Rüdiger ${ }^{2}$, L Ruet ${ }^{15}$, P Russell ${ }^{1}$, K Ryan $^{12}$, S Sakata ${ }^{43}$, M Samidi ${ }^{1}$, L Sancho de la Jordana ${ }^{51}$, V Sandberg ${ }^{12}$, V Sannibale ${ }^{1}$, S Saraf ${ }^{52}$, P Sarin ${ }^{15}$, B S Sathyaprakash ${ }^{28}$,

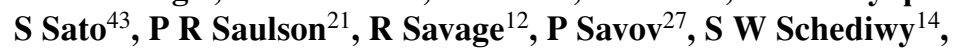
R Schilling $^{2}$, R Schnabel ${ }^{2}$, R Schofield ${ }^{20}$, B F Schutz ${ }^{10,28}$, P Schwinberg ${ }^{12}$, S M Scott ${ }^{34}$, A C Searle ${ }^{34}$, B Sears ${ }^{1}$, F Seifert ${ }^{2}$, D Sellers ${ }^{18}$, A S Sengupta ${ }^{1}$, P Shawhan $^{22}$, D H Shoemaker ${ }^{15}$, A Sibley ${ }^{18}$, X Siemens ${ }^{3}$, D Sigg ${ }^{12}$, S Sinha ${ }^{4}$, A M Sintes ${ }^{10,51}$, B J J Slagmolen ${ }^{34}$, J Slutsky ${ }^{5}$, J R Smith ${ }^{21}$, M R Smith ${ }^{1}$, N D Smith ${ }^{15}$, K Somiya ${ }^{2,10}$, B Sorazu ${ }^{13}$, L C Stein ${ }^{15}$,

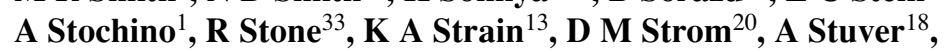
T Z Summerscales ${ }^{53}$, K-X Sun ${ }^{4}$, M Sung , P J Sutton $^{28}$, H Takahashi ${ }^{10}$, D B Tanner ${ }^{6}$, R Taylor ${ }^{1}, \mathbf{R}$ Taylor $^{13}, \mathbf{J}_{\text {Thacker }}{ }^{18}, \mathbf{K}$ A Thorne ${ }^{31}$, K S Thorne ${ }^{27}$, A Thüring ${ }^{9}$, M Tinto ${ }^{1}$, K V Tokmakov ${ }^{13}$, C Torres $^{18}$, C Torrie ${ }^{13}$, G Traylor ${ }^{18}$, M Trias ${ }^{51}$, W Tyler ${ }^{1}$, D Ugolini ${ }^{54}$, J Ulmen ${ }^{4}$, K Urbanek ${ }^{4}$, H Vahlbruch ${ }^{9}$, C Van Den Broeck ${ }^{28}$, M van der Sluys ${ }^{42}$,

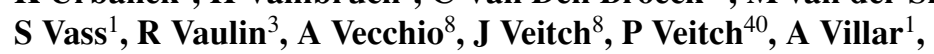
C Vorvick ${ }^{12}$, S P Vyachanin ${ }^{17}$, S J Waldman ${ }^{1}$, L Wallace ${ }^{1}$, H Ward ${ }^{13}$, R Ward ${ }^{1}$, M Weinert ${ }^{2}$, A Weinstein ${ }^{1}, \mathbf{R}$ Weiss $^{15}, \mathbf{S}$ Wen $^{5}$, K Wette $^{34}$, J T Whelan ${ }^{10}$, S E Whitcomb ${ }^{1}$, B F Whiting ${ }^{6}$, C Wilkinson ${ }^{12}$, P A Willems ${ }^{1}$, H R Williams ${ }^{31}$, L Williams ${ }^{6}$, B Willke ${ }^{2,9}$, I Wilmut $^{38}$, W Winkler ${ }^{2}$, C C Wipf ${ }^{15}$, A G Wiseman ${ }^{3}$, G Woan $^{13}$, R Wooley ${ }^{18}$,

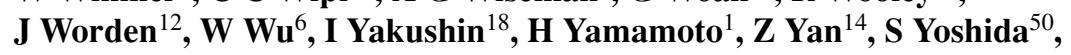
M Zanolin ${ }^{39}$, J Zhang ${ }^{30}$, L Zhang ${ }^{1}$, C Zhao ${ }^{14}$, N Zotov ${ }^{55}$, M Zucker ${ }^{15}$ and J Zweizig ${ }^{1}$ (LIGO Scientific Collaboration) 
${ }^{1}$ LIGO - California Institute of Technology, Pasadena, CA 91125, USA

2 Albert-Einstein-Institut, Max-Planck-Institut für Gravitationsphysik, D-30167 Hannover, Germany

${ }^{3}$ University of Wisconsin-Milwaukee, Milwaukee, WI 53201, USA

${ }^{4}$ Stanford University, Stanford, CA 94305, USA

${ }^{5}$ Louisiana State University, Baton Rouge, LA 70803, USA

${ }^{6}$ University of Florida, Gainesville, FL 32611, USA

${ }^{7}$ Columbia University, New York, NY 10027, USA

${ }^{8}$ University of Birmingham, Birmingham, B15 2TT, UK

${ }^{9}$ Leibniz Universität Hannover, D-30167 Hannover, Germany

${ }^{10}$ Albert-Einstein-Institut, Max-Planck-Institut für Gravitationsphysik,

D-14476 Golm, Germany

11 Carleton College, Northfield, MN 55057, USA

12 LIGO Hanford Observatory, Richland, WA 99352, USA

${ }^{13}$ University of Glasgow, Glasgow, G12 8QQ, UK

${ }^{14}$ University of Western Australia, Crawley, WA 6009, Australia

15 LIGO-Massachusetts Institute of Technology, Cambridge, MA 02139, USA

${ }^{16}$ San Jose State University, San Jose, CA 95192, USA

${ }^{17}$ Moscow State University, Moscow, 119992, Russia

${ }^{18}$ LIGO Livingston Observatory, Livingston, LA 70754, USA

${ }^{19}$ Washington State University, Pullman, WA 99164, USA

${ }^{20}$ University of Oregon, Eugene, OR 97403, USA

${ }^{21}$ Syracuse University, Syracuse, NY 13244, USA

22 University of Maryland, College Park, MD 20742, USA

23 University of Massachusetts, Amherst, MA 01003, USA

${ }^{24}$ NASA/Goddard Space Flight Center, Greenbelt, MD 20771, USA

${ }^{25}$ University of Sannio at Benevento, I-82100 Benevento, Italy

${ }^{26}$ Charles Sturt University, Wagga Wagga, NSW 2678, Australia

${ }^{27}$ Caltech-CaRT, Pasadena, CA 91125, USA

${ }^{28}$ Cardiff University, Cardiff, CF24 3AA, UK

${ }^{29}$ University of Salerno, 84084 Fisciano (Salerno), Italy

${ }^{30}$ University of Michigan, Ann Arbor, MI 48109, USA

31 The Pennsylvania State University, University Park, PA 16802, USA

32 Inter-University Centre for Astronomy and Astrophysics, Pune - 411007, India

${ }^{33}$ The University of Texas at Brownsville and Texas Southmost College,

Brownsville, TX 78520, USA

${ }^{34}$ Australian National University, Canberra, 0200, Australia

35 Southern University and $\mathrm{A} \backslash \& \mathrm{M}$ College, Baton Rouge, LA 70813, USA

${ }^{36}$ California Institute of Technology, Pasadena, CA 91125, USA

${ }^{37}$ University of Rochester, Rochester, NY 14627, USA

${ }^{38}$ Rutherford Appleton Laboratory, Chilton, Didcot, Oxon OX11 0QX, UK

${ }^{39}$ Embry-Riddle Aeronautical University, Prescott, AZ 86301, USA

${ }^{40}$ University of Adelaide, Adelaide, SA 5005, Australia

${ }^{40}$ University of Southampton, Southampton, SO17 1BJ, UK

42 Northwestern University, Evanston, IL 60208, USA

43 National Astronomical Observatory of Japan, Tokyo 181-8588, Japan

${ }^{44}$ Institute of Applied Physics, Nizhny Novgorod, 603950, Russia

${ }^{45}$ University of Strathclyde, Glasgow, G1 1XQ, UK

${ }^{46}$ University of Minnesota, Minneapolis, MN 55455, USA

47 The University of Texas at Austin, Austin, TX 78712, USA

${ }^{48}$ Loyola University, New Orleans, LA 70118, USA

${ }^{49}$ Hobart and William Smith Colleges, Geneva, NY 14456, USA

${ }^{50}$ Southeastern Louisiana University, Hammond, LA 70402, USA

${ }^{51}$ Universitat de les Illes Balears, E-07122 Palma de Mallorca, Spain

52 Sonoma State University, Rohnert Park, CA 94928, USA

53 Andrews University, Berrien Springs, MI 49104, USA

54 Trinity University, San Antonio, TX 78212, USA

55 Louisiana Tech University, Ruston, LA 71272, USA

E-mail: i.heng@physics.gla.ac.uk 
Received 8 August 2008, in final form 6 October 2008

Published 27 November 2008

Online at stacks.iop.org/CQG/25/245008

\begin{abstract}
We present the results of the first joint search for gravitational-wave bursts by the LIGO and GEO 600 detectors. We search for bursts with characteristic central frequencies in the band $768-2048 \mathrm{~Hz}$ in the data acquired between 22 February and 23 March, 2005 (fourth LSC Science Run-S4). We discuss the inclusion of the GEO 600 data in the Waveburst-CorrPower pipeline that first searches for coincident excess power events without taking into account differences in the antenna responses or strain sensitivities of the various detectors. We compare the performance of this pipeline to that of the coherent Waveburst pipeline based on the maximum likelihood statistic. This likelihood statistic is derived from a coherent sum of the detector data streams that takes into account the antenna patterns and sensitivities of the different detectors in the network. We find that the coherent Waveburst pipeline is sensitive to signals of amplitude $30-50 \%$ smaller than the Waveburst-CorrPower pipeline. We perform a search for gravitational-wave bursts using both pipelines and find no detection candidates in the $\mathrm{S} 4$ data set when all four instruments were operating stably.
\end{abstract}

PACS numbers: $04.80 . \mathrm{Nn}, 95.30 . \mathrm{Sf}, 95.85 . \mathrm{Sz}$

(Some figures in this article are in colour only in the electronic version)

\title{
1. Introduction
}

The worldwide network of interferometric gravitational wave detectors currently includes the three detectors of LIGO [1], as well as the GEO 600 [2], Virgo [3] and TAMA300 [4] detectors. The LIGO and GEO 600 detectors and affiliated institutions form the LIGO Scientific Collaboration (LSC). The LSC has performed several joint operational runs of its detectors. During the course of the most recent runs, the detectors have reached sensitivities that may allow them to detect gravitational waves from distant astrophysical sources.

Expected sources of gravitational-wave bursts include, for example, core-collapse supernovae and the merger phase of inspiralling compact object binaries. In general, due to the complex physics involved in such systems, the waveforms of the gravitational wave signals are not well modelled.

There are two broad categories of gravitational-wave bursts searches. Triggered searches use information from an external observation, such as a gamma-ray burst, to focus on a short time interval, permitting a relatively low threshold to be placed on signal-to-noise ratio (SNR) for a fixed false alarm probability. Untriggered searches are designed to maximize the detection efficiency for gravitational-wave bursts for data acquired over the entire run (spanning weeks or months depending on the run) for a given false alarm probability. In general, untriggered searches are designed to scan the entire sky for gravitational-wave bursts though searches performed for a particular sky location (for example, the Galactic centre) can also come under this category. 
Previous untriggered burst searches performed by the LSC typically consisted of a first stage that identifies coincident excess power in multiple detectors and a second stage that tests the consistency of the data with the presence of a gravitational wave signal [5-8]. The Waveburst-CorrPower (WBCP) pipeline is an example of such a two-stage analysis. The first stage, performed by Waveburst, involves a wavelet transformation of the data and identification of excess power in time-frequency volumes that are coincident between multiple detectors [9]. A waveform consistency test is then performed by the CorrPower algorithm which quantifies how well the detected waveforms match each other by using the cross-correlation $r$ statistic $[10,11]$. This approach has been used by the LSC to search for gravitational-wave bursts in LIGO data acquired during the second through fourth Science Runs.

One should note that this pipeline requires coincident excess power to be observed in all detectors in the network to trigger the waveform consistency test performed by CorrPower. Furthermore, CorrPower works on the underlying assumption that all detectors in the network have similar responses to the same gravitational wave signal. This assumption is valid for the LIGO detectors, which have similar antenna patterns. Their strain sensitivities are also similar, though the $2 \mathrm{~km}$ interferometer at Hanford is a factor of two less sensitive than its $4 \mathrm{~km}$ counterparts. On the other hand, GEO 600 has a different orientation on the Earth (see figure 1 and discussion in section 2), so that the received signal in this detector is a different linear combination of the $h_{+}$and $h_{\times}$polarizations from that in the LIGO detectors. Furthermore, the GEO 600 noise spectrum during the fourth LSC Science Run, S4, (22 February to 23 March, 2005) was quite different from those of LIGO (see figure 2), with best GEO 600 sensitivity around $1 \mathrm{kHz}$. As a consequence, the approximation of a common signal response breaks down for the LIGO-GEO network. For example, a low-frequency gravitational-wave burst may appear in LIGO but not be evident in GEO 600. Alternatively, a high-frequency gravitational-wave burst may appear more strongly in GEO 600 than in LIGO if it is incident from a sky direction for which the GEO antenna response is significantly larger than those of the LIGO detectors. These effects complicate a coincidence analysis of the sort employed by the LSC in previous burst searches. Such analyses demand coincident excitation in all detectors in the network. As a result, the sensitivity of the network tends to be limited by the least sensitive detector [8].

Coherent burst search algorithms have been developed to fold in data from a network of detectors with different sensitivities and orientations. Methods for coherent burst searches were first described in [12, 13]. In [12], Gürsel and Tinto have shown for a network of three detectors that a gravitational wave signal can be cancelled out by forming a particular linear combination of data from detectors in the network, producing what is commonly referred to as the null stream. It is now well known [14] that the approach of Gürsel and Tinto is a special case of maximum likelihood inference. In [13], Flanagan and Hughes describe a general likelihood method for the detection and reconstruction of the two polarizations of a gravitational wave signal. A modified likelihood method [15] which uses model-independent constraints imposed on the likelihood functional is implemented in the coherent Waveburst (cWB) algorithm [16]. It uses the maximum likelihood statistic, calculated for each point in the sky, which represents the total signal-to-noise ratio of the gravitational wave signal detected in the network. Coincident instrumental or environmental transient artefacts (glitches) that are unlikely to be consistent between the detectors will usually leave some residual signature in the null stream, which can be used as a powerful tool for rejection of glitches $[17,18]$. Recently it was shown that straightforward application of the maximum likelihood method to searches of bursts with unknown waveforms can lead to inconsistencies and unphysical results $[15,19]$. All these problems occur due to the rank deficiency of the network response matrix and therefore can be cured by a suitable regularization procedure [14]. 

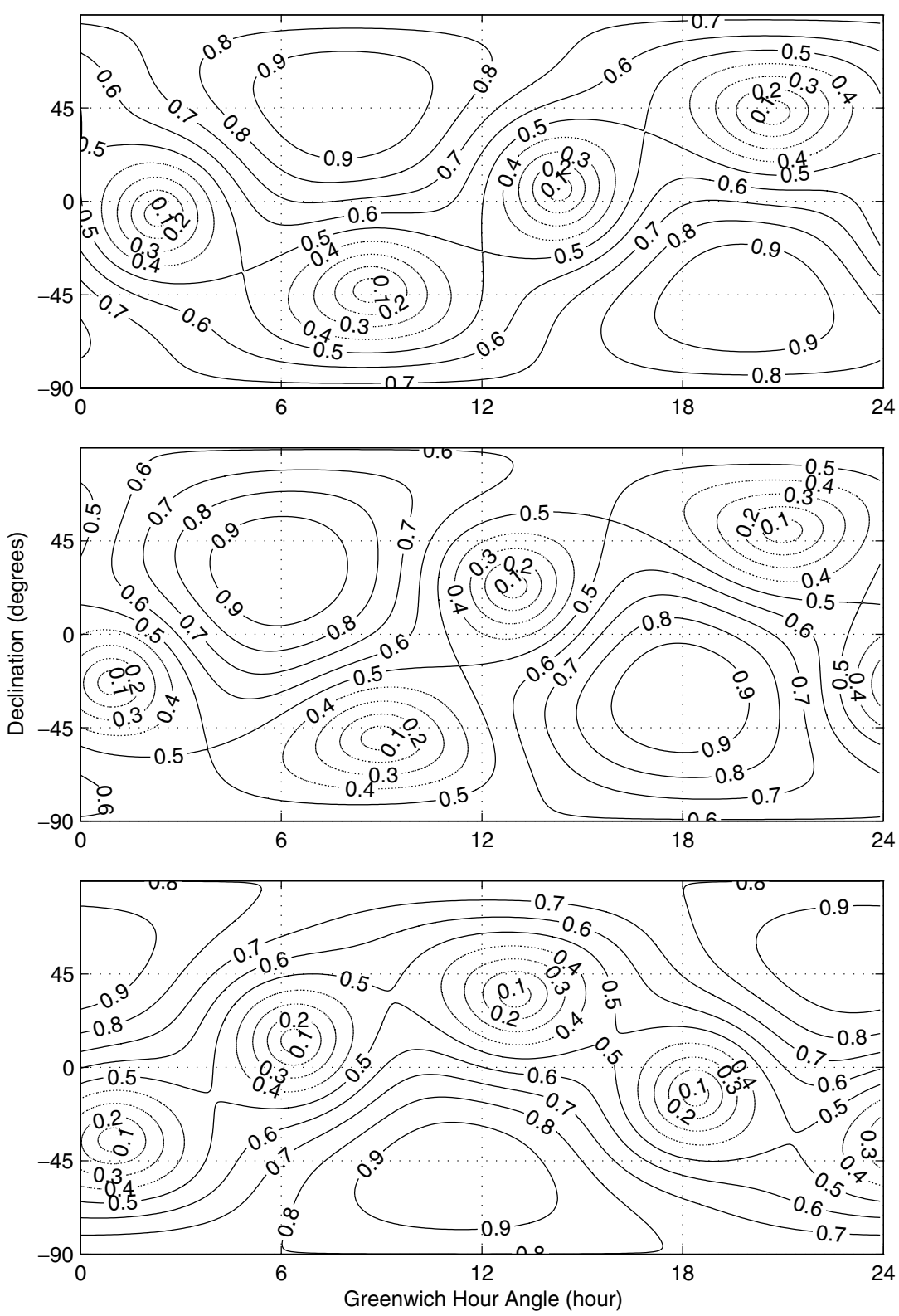

Figure 1. Antenna patterns $\left(F_{+}^{2}+F_{\times}^{2}\right)$ of the Hanford (top), Livingston (middle) and GEO 600 (bottom) detectors. The locations of the maxima and minima in the antenna patterns for Hanford and Livingston are close. However, the antenna pattern for GEO 600 is different from those of the LIGO detectors.

In this paper, we present the first burst search using data from the three LIGO detectors and GEO 600, acquired during the fourth Science Run of the LSC. We present a search for gravitational-wave bursts between 768 and $2048 \mathrm{~Hz}$ using both the Waveburst-CorrPower and coherent Waveburst pipelines. We begin with a brief description of the detectors in section 2 


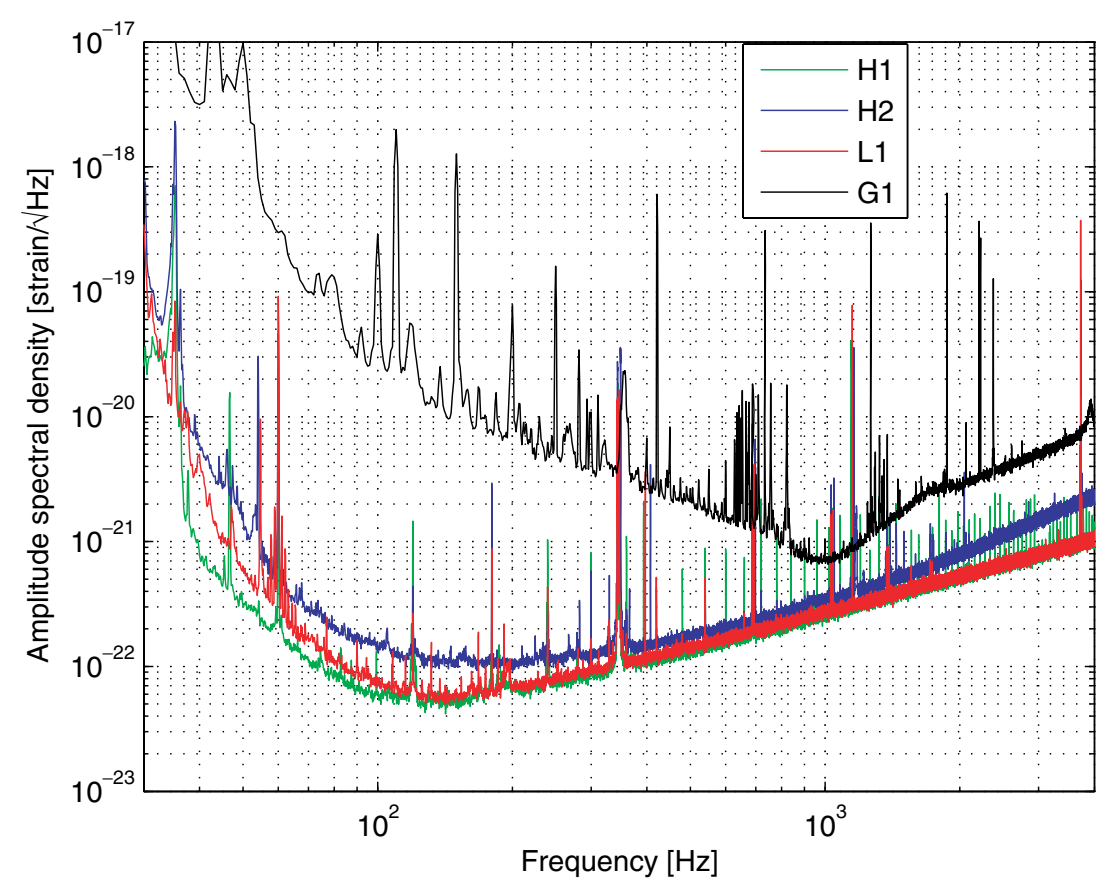

Figure 2. Strain spectral densities of the LIGO Hanford $2 \mathrm{~km}$ and $4 \mathrm{~km}$ detectors $(\mathrm{H} 1, \mathrm{H} 2)$ and the LIGO Livingston detector (L1) as well as the GEO 600 detector (G1) during the S4 run. The plotted strain sensitivity curves are the best for the LIGO detectors, obtained on 26 February, 2005, for $\mathrm{H} 1$ and $\mathrm{H} 2$ and 11 March, 2005, for L1. The GEO 600 sensitivity curve is typical of the detector's performance during the S4 run.

before describing the two methods used to analyse the acquired data in section 3 . We then detail the additional selection criteria and vetoes in section 4 . We present the results of the search in section results and compare the detection efficiencies of the two methods. Finally, we discuss our observations in section 6.

\section{Instruments and data}

Here, we present a brief description of the main features of the LIGO and GEO 600 detectors. A more detailed description of the LIGO detectors in their S4 configuration can be found in [1]. The most recent description of the GEO 600 detector can be found in [2, 20].

LIGO consists of three laser interferometric detectors at two locations in the United States of America. There are two detectors at the Hanford site, one with $4 \mathrm{~km}$ arms and another with $2 \mathrm{~km}$ arms, which we refer to as $\mathrm{H} 1$ and $\mathrm{H} 2$, respectively. In Livingston, there is one detector with $4 \mathrm{~km}$ arms which we refer to as L1. Each detector consists of a Michelson interferometer with Fabry-Perot cavities in both arms. The laser light power builds up in these resonant cavities, enhancing the sensitivity of the detector. At the input to the interferometer, there is a power-recycling mirror which increases the stored laser light power in the interferometer. This reduces the effect of shot noise, allowing for better sensitivity at higher frequencies. 
The GEO 600 laser interferometric gravitational wave detector has been built and operated by a British-German collaboration. It is located near Hannover in Germany and, along with the three LIGO detectors, is part of the LSC interferometer network. GEO 600 is a Michelson interferometer with six hundred metre arms. The optical path is folded once to give a $2400 \mathrm{~m}$ round-trip length. To compensate for the shorter arm length, GEO 600 incorporates not only power-recycling, but also signal-recycling (SR), which allows the response of the interferometer to be shaped, and the frequency of maximum response to be chosen-the 'SR detuning' frequency. During the S4 run, a test power-recycling mirror with $1.35 \%$ transmission was installed, yielding an intra-cavity power of only $500 \mathrm{~W}$. As a result, the sensitivity of GEO 600 above $500 \mathrm{~Hz}$ was limited nearly entirely by shot noise [21]. The SR mirror had about $2 \%$ transmission and the SR detuning frequency was set at $1 \mathrm{kHz}$. An overview of the signal processing and the calibration process in S4 is given in [22].

The strain spectral densities of each detector during S4 are shown in figure 2. The duty factor indicates the percentage of time each detector was operational during the S4 run. GEO 600 achieved a duty factor of $96.5 \%$, despite running in a fully automated mode with minimal human intervention for operation and maintenance. $\mathrm{H} 1, \mathrm{H} 2$ and $\mathrm{L} 1$ achieved duty factors of $80.5 \%, 81.4 \%$ and $74.5 \%$ respectively. To calibrate the LIGO and GEO 600 detectors, continuous sinusoidal signals are injected into the actuation signals of some mirrors at several frequencies. The resulting displacement is known and used to determine the transfer function of the detector to an incoming gravitational wave, with an accuracy conservatively estimated at $10 \%[23,24]$. For GEO 600 , the demodulated signal from the main photodetector is recombined using a maximum likelihood method [25].

The strain detectable at each detector, $h(t)$, for a GW signal with strain amplitudes of $h_{+}(t)$ and $h_{\times}(t)$ in the plus and cross polarizations, respectively, is given by

$$
h(t)=F_{+}\left(H_{\text {Greenwich }}, \delta, \psi\right) h_{+}(t)+F_{\times}\left(H_{\text {Greenwich }}, \delta, \psi\right) h_{\times}(t)
$$

where $F_{+}\left(H_{\text {Greenwich }}, \delta, \psi\right)$ and $F_{\times}\left(H_{\text {Greenwich }}, \delta, \psi\right)$ are the antenna responses to the plus and cross-polarizations. The antenna responses depend on the locations and orientations of the interferometers on the Earth's surface, where $H_{\text {Greenwich }}$ and $\delta$ are the Greenwich hour angle and declination of the source in Earth-centred coordinates and $\psi$ is the polarization angle (see [26] for an explicit definition).

Figure 1 shows the sum-squared antenna response $\left(F_{+}^{2}+F_{\times}^{2}\right)$ for each site in the LIGOGEO network in a fixed-Earth coordinate system. The Hanford and Livingston detectors are well aligned to each other and, therefore, have very similar antenna patterns. On the other hand, the GEO 600 detector has different antenna patterns, with peak sensitivities in sky locations that are near the minima of the LIGO detectors.

\section{Search algorithms}

In this section, we describe the two search pipelines used for the analysis. The WBCP pipeline is almost identical to that used to perform previous searches for gravitational-wave bursts [5-7]. However, for the analysis reported in this paper, Waveburst is applied to data acquired by the LIGO and GEO 600 detectors, while CorrPower is applied only to data acquired by the LIGO detectors (see below for further explanation). The performance of the WBCP pipeline will be compared to that of the cWB pipeline. The same data were processed using the two pipelines. 


\subsection{Waveburst and CorrPower pipeline}

We give a brief description of the WBCP pipeline. More detailed descriptions of the Waveburst $^{56}$ and CorrPower ${ }^{57}$ algorithms can be found in $[9,10]$ respectively.

The data acquired by each detector in the network are processed by the Waveburst algorithm which performs a wavelet transformation using the Meyer wavelet [27] This creates a time-frequency (TF) map of the data. A threshold is applied to this map to select TF volumes or pixels with significant excess power. As with previous LSC GW burst searches, this threshold is set such that the loudest $10 \%$ of the TF pixels are selected. Coincident excess power pixels from multiple detectors are then clustered together to form coincident triggers and an overall significance, $Z_{\mathrm{g}}$, is assigned to the coincident pixel cluster [7].

The central time and duration of these triggers are then passed on to CorrPower. CorrPower calculates the cross-correlation statistic, commonly denoted by $r$, for the time series data from a pair of detectors in the following manner:

$$
r=\frac{\sum_{i=1}^{N}\left(x_{i}-\bar{x}\right)\left(y_{i}-\bar{y}\right)}{\sqrt{\sum_{i=1}^{N}\left(x_{i}-\bar{x}\right)^{2}} \sqrt{\sum_{i=1}^{N}\left(y_{i}-\bar{y}\right)^{2}}},
$$

where $x_{i}$ and $y_{i}$ are the $i$ th data sample from the two time series from the detector pair, with $\bar{x}$ and $\bar{y}$ their respective means. The total number of samples over which $r$ is calculated is denoted by $N$. This quantity is calculated for a range of time shifts, corresponding to the range of possible light travel time differences between the detectors for gravitational waves incident from different directions (up to $\pm 10 \mathrm{~ms}$ for the LIGO detectors). The CorrPower algorithm effectively quantifies how well the data from different detectors match, thereby performing an approximate waveform consistency test.

A Kolmogorov-Smirnov test is used to compare the distribution of the $r$ statistic with a normal distribution with zero mean and a variance equal to the inverse of the number of data samples in the time series. For coincident excess power in multiple detectors, we expect the $r$ statistic distribution to be inconsistent with a normal distribution, so we calculate the confidence

$$
C=-\log _{10}(S)
$$

where $S$ is the statistical significance of the $r$ statistic deviation from the normal distribution [11]. The overall confidence, $\Gamma$, is calculated by taking the average confidence for all detector pairs

$$
\Gamma=\frac{1}{N_{\text {pairs }}} \sum_{k=1}^{N_{\text {pairs }}} C_{k},
$$

where $N_{\text {pairs }}$ is the total number of detector pairs in the network (for LIGO, $N_{\text {pairs }}=3$, for LIGO-GEO, $N_{\text {pairs }}=6$ but only the three LIGO pairs are used here) and $C_{k}$ is the measured confidence for the $k$ th detector pair.

The use of CorrPower in this pipeline is best suited to detectors that are closely aligned, such as the LIGO detectors, since it relies on the detector responses to incoming gravitational waves to be correlated. Because GEO 600 is not aligned with the LIGO detectors, an $r$ statistic calculated for the full LIGO-GEO network would be small for some sky locations and polarizations for which the detected signal in GEO 600 has little or no correlation with the

56 The version of Waveburst used for this analysis may be found at http://ldas-sw.ligo.caltech.edu/cgi-bin/ cvsweb.cgi/Analysis/WaveBurst/S4/?cvsroot=GDS with the CVS tag 'S4'.

57 The version of CorrPower used for this analysis may be found at http://www.lsc-group.phys.uwm.edu/cgi-bin/ cvs/viewcvs.cgi/matapps/src/searches/burst/CorrPower/?cvsroot=lscsoft with the CVS tag 'CorrPower-080605'. 
detected signal in LIGO. This can be accounted for if the source location and signal waveform are known, but for an all-sky burst search, we find that including GEO 600 in the $r$-statistic calculation has little or no benefit. Therefore, we chose to apply CorrPower to only the LIGO subset of detectors.

The search pipeline also performs two diagnostic tests on times when $\mathrm{H} 1$ and $\mathrm{H} 2$ Waveburst triggers are coincident. These two tests take advantage of the fact that $\mathrm{H} 1$ and $\mathrm{H} 2$ are located in the same site and are fully aligned. As a consequence, true gravitational wave signals in $\mathrm{H} 1$ and $\mathrm{H} 2$ should be strongly correlated and have the same strain amplitude. The pipeline requires, therefore, the $\mathrm{H} 1-\mathrm{H} 2$ triggers to have amplitude ratios greater than 0.5 and less than 2 (this range is determined by studying the amplitude ratios of simulated gravitational wave signals added to the $\mathrm{H} 1$ and $\mathrm{H} 2$ data streams) [7]. CorrPower also calculates the sign of the cross-correlation between $\mathrm{H} 1$ and $\mathrm{H} 2$ with no relative time delay, $R_{0}$, and demands that this quantity be positive.

\subsection{Coherent Waveburst}

The cWB pipeline ${ }^{58}$ uses the regularized likelihood method for the detection of gravitationalwave bursts in interferometric data [15]. This pipeline is designed to work with arbitrary networks of gravitational wave interferometers. Like the WBCP pipeline described in the previous section, the cWB pipeline performs analysis in the wavelet domain. Both pipelines use the same data conditioning algorithms, but the generation of burst triggers is different. The WBCP pipeline performs TF coincidence of the excess power triggers between the detectors. The cWB pipeline combines the individual detector data streams into a coherent likelihood statistic.

3.2.1. Regularized likelihood. In the presence of a gravitational wave the whitened network output in the wavelet domain is

$$
\mathbf{w}=\mathbf{f}_{+} h_{+}+\mathbf{f}_{\times} h_{\times}+\mathbf{n} .
$$

Here the vectors $\mathbf{f}_{+}$and $\mathbf{f}_{\times}$characterize the network sensitivity to the two polarization components $h_{+}$and $h_{\times}$, and $\mathbf{n}$ is the noise vector. At each time-frequency pixel $[i, j]$, the whitened network output is

$$
\mathbf{w}=\left(\frac{a_{1}[i, j]}{\sigma_{1}[i, j]}, \ldots, \frac{a_{K}[i, j]}{\sigma_{K}[i, j]}\right)
$$

where $a_{1}, \ldots, a_{K}$ are the sampled detector amplitudes in the wavelet domain, $[i, j]$ are their time-frequency indices and $K$ is the number of detectors in the network. Note that the amplitudes $a_{k}$ take into account the time delays of a GW signal incoming from a given point in the sky. In the cWB analysis, we assume that the detector noise is Gaussian and quasistationary. The noise is characterized by its standard deviation $\sigma_{k}[i, j]$ and may vary over the time-frequency plane. The antenna pattern vectors $\mathbf{f}_{+}$and $\mathbf{f}_{\times}$are defined as follows:

$$
\mathbf{f}_{+(\times)}=\left(\frac{F_{1+(\times)}}{\sigma_{1}[i, j]}, \ldots, \frac{F_{K+(\times)}}{\sigma_{K}[i, j]}\right) .
$$

We calculate the antenna pattern vectors in the dominate polarization frame [15], where we call them $f_{1}$ and $f_{2}$. In this frame, they are orthogonal to each other: $\left(\mathbf{f}_{\mathbf{1}} \cdot \mathbf{f}_{\mathbf{2}}\right)=0$. The maximum log-likelihood ratio statistic is calculated as

$$
L=\sum_{i, j \in \Omega_{T F}} \mathbf{w} P \mathbf{w}^{T}, \quad P_{n m}=e_{1 n} e_{1 m}+e_{2 n} e_{2 m}
$$

\footnotetext{
${ }^{58}$ The version of coherent Waveburst used for this analysis maybe found at http://www.ldas-sw.ligo.caltech.edu/
} cgi-bin/cvsweb.cgi/Analysis/WaveBurst/S4/coherent/wat/?cvsroot=GDS with the CVS tag 'S4_LIGO-GEO'. 
where the time-frequency indices $i$ and $j$ run over some area $\Omega_{T F}$ on the TF plane selected for the analysis (network trigger) and the matrix $P$ is a projection constructed from the unit vectors $\mathbf{e}_{\mathbf{1}}$ and $\mathbf{e}_{\mathbf{2}}$ along the directions of $\mathbf{f}_{\mathbf{1}}$ and $\mathbf{f}_{\mathbf{2}}$, respectively. The null space of the projection $P$ defines the reconstructed detector noise which is often called the null stream. The null energy $N$ is calculated by

$$
N=E-L
$$

where

$$
E=\sum_{i, j \in \Omega_{T F}}|\mathbf{w}|^{2},
$$

and $|\mathbf{w}|$ is the vector norm of $\mathbf{w}$. The null energies $N_{k}$ for individual detectors can be also reconstructed [15]. We also introduce a correlated energy $E_{\mathrm{c}}$ which is defined as the sum of the likelihood terms corresponding to the off-diagonal elements of the matrix $P$.

However, the projection $P$ may not always be constructed. For example, for a network of aligned detectors $\left|\mathbf{f}_{2}\right|=0$ and the unity vector $\mathbf{e}_{2}$ is not defined. As shown in [15] even for misaligned detectors the network may be much less sensitive to the secondary GW component $\left(\left|\mathbf{f}_{2}\right| \ll\left|\mathbf{f}_{1}\right|\right)$ and it may not be reconstructed from the noisy data. In order to solve this problem, we introduce a regulator by changing the norm of the $\mathbf{f}_{\mathbf{2}}$ vector

$$
\left|\mathbf{f}_{\mathbf{2}}^{\prime}\right|^{2}=\left|\mathbf{f}_{\mathbf{2}}\right|^{2}+\delta\left(\left|\mathbf{f}_{\mathbf{1}}\right|^{2}-\left|\mathbf{f}_{\mathbf{2}}\right|^{2}\right)
$$

where the parameter $\delta$ is selected to be 0.1 . The regularized likelihood is then calculated by using the operator $P$ constructed from the vectors $\mathbf{e}_{\mathbf{1}}$ and $\mathbf{e}_{2}^{\prime}$, where $\mathbf{e}_{2}^{\prime}$ is $\mathbf{f}_{\mathbf{2}}$ normalized by $\left|\mathbf{f}_{\mathbf{2}}^{\prime}\right|$. All other coherent statistics, such as the null and correlated energies, are calculated accordingly.

3.2.2. Reconstruction of network triggers. Coherent Waveburst first resamples the calibrated data streams to $4096 \mathrm{~Hz}$ before whitening them in the wavelet domain. The Meyer wavelet is used to produce time-frequency maps with the frequency resolutions of $8,16,32,64,128$ and $256 \mathrm{~Hz}$. An upper bound on the total energy $|\mathbf{w}|^{2}$ is then calculated for each network pixel; if greater than a threshold, the total energy is then computed for each of 64800 points in the sky placed in a grid with $1^{\circ} \times 1^{\circ}$ resolution. If the maximum value of $|\mathbf{w}|^{2}$ is greater than $12-13$ (depending on the frequency resolution), the network pixel is selected for likelihood analysis. The selected pixels are clustered together to form network triggers [16].

After the network triggers are identified, we reconstruct their parameters, including the two GW polarizations, the individual detector responses and the regularized likelihood triggers. All the trigger parameters are calculated for a point in the sky which is selected by using a criteria based on the correlated energy and null energy. Namely, we select such a point in the sky where the network correlation coefficient $c c$ is maximized:

$$
c c=\frac{E_{\mathrm{c}}}{N+E_{\mathrm{c}}} .
$$

For a GW signal at the true source location a small null energy and large correlated energy is expected with the value of $c c$ close to unity.

The identification of the network triggers and reconstruction of their parameters is performed independently for each frequency resolution. As a result multiple triggers at the same time-frequency area may be produced. The trigger with the largest value of the likelihood in the group is selected for the post-production analysis. 
3.2.3. Post-production analysis. During the cWB post-production analysis, we apply additional selection cuts in order to reject instrumental and environmental artefacts. For this we use coherent statistics calculated during the production stage. Empirically, we found the following set of the trigger selection cuts that perform well on the S4 LIGO-GEO data.

Similar to the regularized likelihood statistic, one can define the sub-network likelihood ratios $L_{k}$ where the energy of the reconstructed detector responses is subtracted from $L$ :

$$
L_{k}=L-\left(E_{k}-N_{k}\right),
$$

where

$$
E_{k}=\sum_{i, j \in \Omega_{T F}} w_{k}^{2}[i, j],
$$

and $w_{k}[i, j]$ are the components of the whitened data vector defined by equation (6). In the post-production analysis we require that all $L_{k}$ are greater than 36 which effectively removes single-detector glitches.

Another very efficient selection cut is based on the network correlation coefficient $c c$ and the rank SNR $\rho_{k}$. Typically, for glitches, little correlated energy is detected by the network and the reconstructed detector responses are inconsistent with the detector outputs, which result in a large null energy: $E_{\mathrm{c}}<N$ and $c c \ll 1$. For a gravitational wave signal, we expect $E_{\mathrm{c}}>N$ and the value of $c c$ to be close to unity. We define the effective rank SNR as

$$
\rho_{\mathrm{eff}}=\left(\frac{1}{K} \sum_{k=1}^{K} \rho_{k}^{2}\right)^{c c / 2},
$$

where $\rho_{k}$ is the non-parametric signal-to-noise ratio for each detector based on the pixel rank statistic [28]

$$
y_{k}[i, j]=-\ln \left(\frac{R_{k}[i, j]}{M}\right) .
$$

In the equation above, $R_{k}[i, j]$ is the pixel rank (with $R=1$ for the loudest pixel) and $M$ is the number of pixels used in the ranking process. The statistic $y_{k}[i, j]$ follows an exponential distribution, independent of the underlying distribution of the pixel amplitudes, $w_{k}[i, j]$. The $y_{k}[i, j]$ can be mapped into rank amplitudes $x_{k}[i, j]$ which have Gaussian distribution with unity variance. The $\rho_{k}$ is calculated as the square root of the sum of $x_{k}^{2}[i, j]$ over the pixels in the cluster and it is a robust measure of the SNR of detected events in the case of non-Gaussian detector noise. We place a threshold on $\rho_{\text {eff }}$ to achieve the false alarm rate desired for the analysis.

\section{Data quality}

Spurious excitations caused by environmental and instrumental noise increase the number of background triggers in gravitational-wave burst searches. Periods when there are detector hardware problems or when the ambient environmental noise level is elevated are flagged and excluded from the analysis. These data quality flags are derived from studies of diagnostic channels and from entries made in the electronic logbook by interferometer operators and scientists on duty that indicate periods of anomalous behaviour in the detector. Additionally, we veto times when data triggers are observed in coincidence with short-duration instrumental or environmental transients.

To maximize our chances of detecting a gravitational-wave burst, we must balance the reduction of each detector's observation time due to data quality flags and vetoes against the 
effectiveness for removing background triggers from the analysis. The data quality flags and vetoes for the LIGO and GEO detectors are outlined below. Out of the 334 hours of quadruple coincidence observation time, 257 hours remained after excluding periods flagged by the data quality flags. This observation time is common to both pipelines. The total livetime of data analysed by cWB is larger by $1 \%$ because of different processing of data segments.

\subsection{GEO 600}

4.1.1. Data quality flags. GEO 600 data quality flags include periods when the data acquisition system is saturated (overflow) and when the $\chi^{2}$ value is too high, as explained below.

The GEO 600 data stream is calibrated into a time series representing the equivalent gravitational wave strain at each sample. The GEO 600 calibration process determines if the noise, as measured by the acquired data, is close to that expected from the optical transfer function by using the $\chi^{2}$ statistic [21]. If the $\chi^{2}$ values are too high, it means that the calibration is not valid. Therefore, the $\chi^{2}$ values from the calibration process are an indicator of data quality.

4.1.2. Excess glitches. During the first ten days of the S4 run, one of the suspended GEO components came into contact with a nearby support structure. This caused GEO data to be glitching excessively between 22 February and 4 March, 2005. The glitch rate fluctuated dramatically over this period because the distance between the component and the support structure changed as a function of temperature. Given the large variability in the glitch rate (about one order of magnitude on a timescale of hours), we decided to exclude this period from the analysis.

\section{2. $L I G O$}

The data quality flags and auxiliary-channel vetoes used with the LIGO detectors are explained in more detail in [7]. Basic data quality cuts are first applied to LIGO data segments so as to exclude periods when the detector is out of lock or when simulated GW signals are injected into the detector. Additionally, data segments are excluded from the analysis when there clearly are problems with the LIGO hardware or when environmental noise sources cause spurious transient noise in the data.

We rejected periods when injected sinusoidal signals used for calibration were not present due to problems in the injection hardware. Since the calibration was unknown for these periods, totalling 1203 seconds, the data were excluded from the analysis. A study based on single-detector triggers showed correlations between the loudest triggers and the speeds of local winds. This was most prominent in $\mathrm{H} 2$. Therefore, data were not included in the analysis when the wind speed at the Hanford site was greater than $56 \mathrm{~km} \mathrm{~h}^{-1}$ (35 miles per hour). This excluded a total of 10303 seconds of four-detector livetime. Seismic activity between 0.4 and $2.4 \mathrm{~Hz}$ was observed to cause transients in the detector noise. Excess coincidences were observed between $\mathrm{H} 1$ and $\mathrm{H} 2$ when there was elevated seismic activity in this frequency range. As a result, time intervals when the root-mean-squared seismic signal exceeded seven times its median value were excluded from the analysis. This accounted for 11704 seconds of the four-detector livetime. Correlations were also observed between singledetector triggers and times when data overflows occurred in an analog-digital-converter (ADC) in the length sensing and control subsystem. A data quality flag for the data overflows excluded 10169 seconds of four-detector livetime. Transient dips in the stored light in the arm cavities 
were found to be strongly correlated with periods of high single-detector rates. Data were excluded from the analysis when the change in measured light relative to the last second was greater than $5 \%$ for $\mathrm{H} 2$ and $\mathrm{L} 1$. A threshold of $4 \%$ relative change was used for $\mathrm{H} 1$.

In addition to the exclusion of data segments, triggers attributed to short-duration instrumental or environmental artefacts are excluded from the analysis. This is done by applying vetoes based on triggers generated from auxiliary channels found to be in coincidence with transients in the gravitational wave data, where veto effectiveness (efficiency versus deadtime) is evaluated on time-shifted background data samples prior to use.

\section{Results}

Here we present and compare the results of the WBCP and cWB pipelines applied to the LIGO and GEO 600 data.

A total of 257 hours of quadruple coincidence data were processed with both the WBCP and $\mathrm{cWB}$ pipelines to produce lists of coincident triggers, each characterized by a central time, duration, central frequency and bandwidth. In addition to these characteristics, each trigger also has an estimated significance with respect to the background noise. Waveburst calculates the overall significance, $Z_{\mathrm{g}}$, while CorrPower calculates the confidence, $\Gamma$. For coherent Waveburst, each trigger is characterized by the likelihood and effective SNR (see equations (13) and (15) respectively). Although WBCP calculates $\Gamma$ using only the LIGO detectors, for convenience, we will refer to coincident triggers from either pipeline as quadruple coincidence triggers. The name is still valid for WBCP triggers since the Waveburst stage of the pipeline requires coincident excess power in all four detectors in the network.

The central frequencies for triggers from both pipelines were restricted to lie between 768 and $2048 \mathrm{~Hz}$. This is because the sensitivity of the GEO 600 detector is closest to the LIGO detectors in this frequency range (see figure 2). Moreover, the noise of GEO 600 is not very stationary at frequencies below $500 \mathrm{~Hz}$, and many spurious glitches can be observed in the acquired data. CorrPower computes the $r$ statistic over a broader band (64-3152 Hz), using only LIGO data.

For both pipelines, the L1 data are shifted with respect to H1, H2 and G1 data by one hundred 3.125-second time steps. The applied time shift is sufficiently large that any short gravitational-wave bursts present in the data cannot be observed in coincidence in all detectors. Therefore, we can study the statistics of the noise and tune the thresholds of the pipeline without bias from any gravitational wave signals that might be present in the data. The goal of the tuning is to reduce the number of time-shifted coincidences (background triggers) while maintaining high detection efficiency for simulated gravitational wave signals.

The efficiency of the pipeline at detecting gravitational-wave bursts for the selected thresholds is determined by adding into the data simulated gravitational wave signals of various morphologies and amplitudes. For this study, we used sine-Gaussians, sine waves with a Gaussian envelope, given in the Earth-fixed frame by

$$
\begin{aligned}
& h_{+}(t)=h_{0} \sin \left(2 \pi f_{0}\left[t-t_{0}\right]\right) \exp \left[-\left(2 \pi f_{0}\left[t-t_{0}\right]\right)^{2} / 2 Q^{2}\right], \\
& h_{\times}(t)=0,
\end{aligned}
$$

where $t_{0}$ and $h_{0}$ are the peak time and amplitude of the envelope, $Q$ is the width of the envelope and $f_{0}$ is the central frequency of the signal. The antenna responses (see equation (1)) are generated for each simulated signal assuming a uniform distribution 


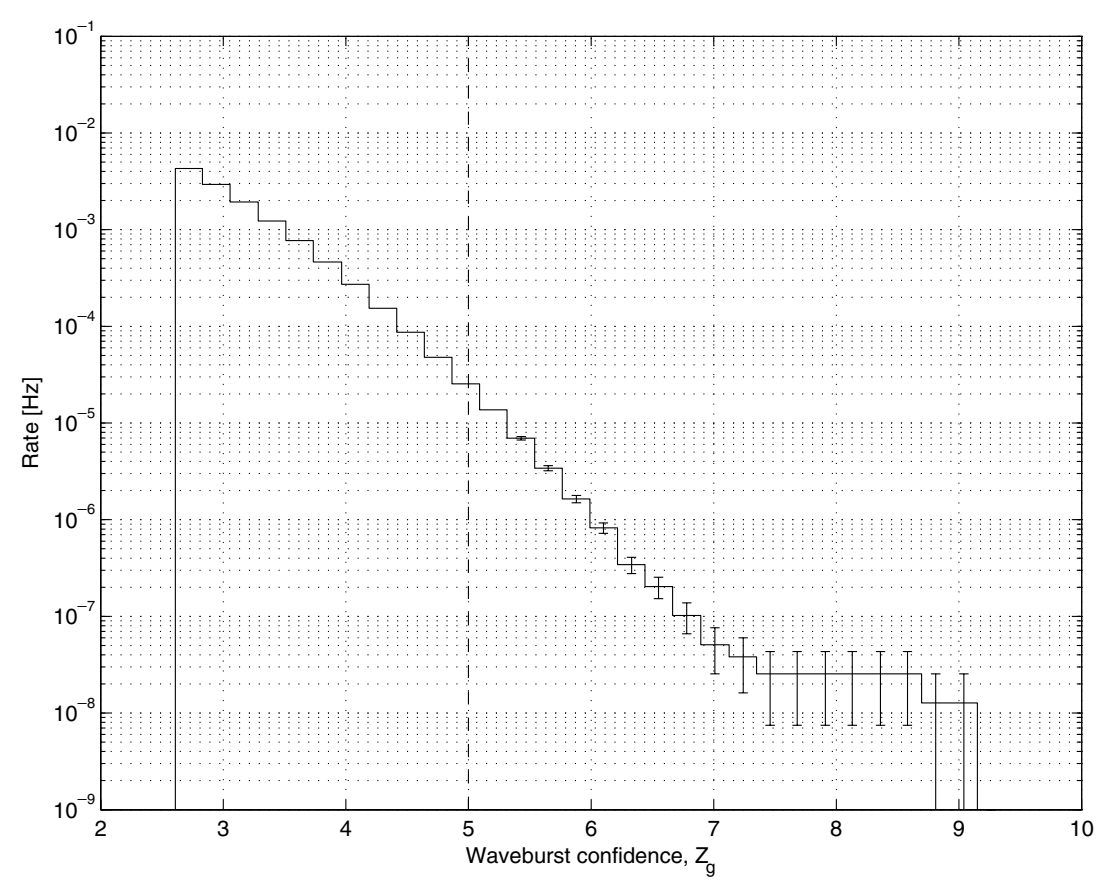

Figure 3. Quadruple coincidence rate as a function of the threshold on the Waveburst significance, $Z_{\mathrm{g}}$. The threshold used for this analysis is indicated by the dashed line. The error bars indicate the range corresponding to $\pm \sqrt{n} / T$, where $n$ is the number of triggers observed above the $Z_{\mathrm{g}}$ threshold over the livetime $T$.

in the sky and a polarization angle $\psi$ uniformly distributed on $[0, \pi]$. The signal strength is parameterized in terms of the root-sum-squared amplitude of the signal, $h_{\mathrm{rss}}$,

$$
h_{\mathrm{rss}} \equiv \sqrt{\int\left(\left|h_{+}(t)\right|^{2}+\left|h_{\times}(t)\right|^{2}\right) \mathrm{d} t} .
$$

The detection efficiency is the fraction of injected signals that produce triggers surviving the selected thresholds for the respective pipeline. We characterize the sensitivity of each pipeline by its $h_{\mathrm{rss}}^{50 \%}$, which is the $h_{\mathrm{rss}}$ at which $50 \%$ of the injected signals are observed at the end of the pipeline (detection efficiency).

\subsection{Waveburst-CorrPower analysis}

For the WBCP pipeline, there are two threshold values to select. The background quadruple coincidence rate as a function of the threshold on Waveburst significance is shown in figure 3. Since the calculation of the $r$-statistic by CorrPower is computationally expensive and time consuming, we reduce the number of triggers by selecting a Waveburst significance threshold of $Z_{\mathrm{g}}=5$, for a false alarm rate of approximately $3 \times 10^{-5} \mathrm{~Hz}$.

The CorrPower confidence, $\Gamma$, is then calculated for each surviving trigger. A scatter plot of $\Gamma$ versus $Z_{\mathrm{g}}$ for these triggers can be seen in figure 4(a). Note that all triggers have $\Gamma$ values less than 4 . The distributions of the $\Gamma$ values of both the time-shifted background triggers and the unshifted triggers are plotted in figure $4(b)$. 
(a)

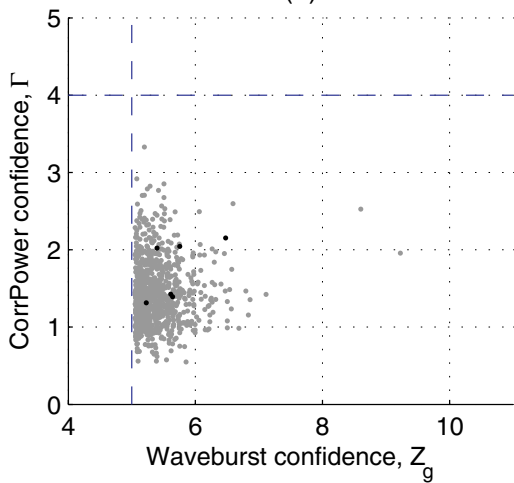

(b)

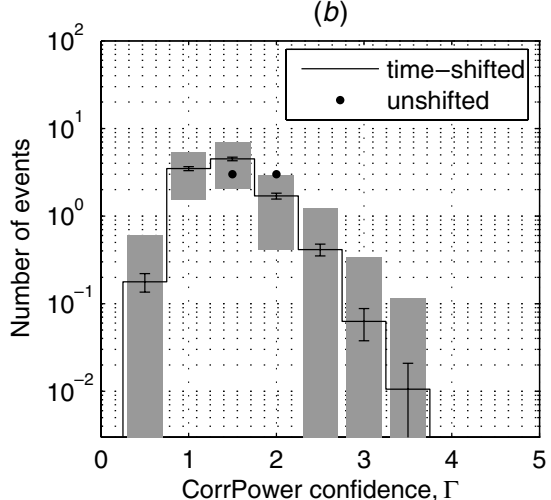

Figure 4. (a) Scatterplot of $r$-statistic confidence, $\Gamma$, versus Waveburst $Z_{\mathrm{g}}$. The time-shifted background triggers are plotted as grey dots while the unshifted triggers are plotted as black dots. The dashed line indicates the $\Gamma$ threshold chosen for this analysis. $(b)$ Overlaid histograms of the unshifted triggers and the $\Gamma$ distribution for the time-shifted triggers averaged over the 100 time shifts. The grey patches indicate the standard deviation in the number of triggers at each time shift. The error bars indicate the range corresponding to $\pm \sqrt{n} / 100$, where $n$ is the total number of triggers in each bin.

Table 1. Table of background triggers and $h_{\mathrm{rss}}^{50 \%}$ as a function of $\Gamma$. The total number of background triggers observed over all 100 time-shifts is shown.

\begin{tabular}{lllll}
\hline & \multirow{2}{*}{$\begin{array}{l}\text { Number of } \\
\text { background triggers }\end{array}$} & \multicolumn{3}{c}{$h_{\mathrm{rss}}^{50 \%}\left[\times 10^{-21} \mathrm{~Hz}^{-1 / 2}\right]$} \\
\cline { 3 - 5 } & $f=849 \mathrm{~Hz}$ & $1053 \mathrm{~Hz}$ & $1615 \mathrm{~Hz}$ \\
\hline 0 & 881 & 6.6 & 6.9 & 13.5 \\
3 & 1 & 6.6 & 7.1 & 13.7 \\
4 & 0 & 6.8 & 7.2 & 13.9 \\
\hline
\end{tabular}

Table 1 shows the number of background coincidences and the $h_{\mathrm{rss}}^{50 \%}$ values for sineGaussian injections of different central frequencies for several trial values of the threshold on $\Gamma$ : $\Gamma>0$ (CorrPower not used), $\Gamma>3$ and $\Gamma>4$. We note that the $h_{\mathrm{rss}}^{50 \%}$ values for a threshold of $\Gamma=4$ are only a few percent higher than those for a threshold of $\Gamma=3$, while the number of background triggers is reduced from 1 to 0 . With the implied reduction rate in false alarm rate in mind, we choose the CorrPower threshold of $\Gamma=4$.

The fraction of sine-Gaussian signals detected above threshold (detection efficiency) as a function of injected $h_{\text {rss }}$ is shown in figure 5. Note that the detection efficiencies do not reach 1 for even the loudest injected signals because of the application of auxiliary-channel vetoes. This effect was also observed in [7]. The detector is effectively blind to GW for the duration of the veto because we are excluding any observations within this period. This exclusion means that there is a non-zero false dismissal probability, even for the loudest GW signals.

\subsection{Coherent Waveburst analysis}

For cWB, the tuning strategy is to set thresholds such that no background triggers are observed. We first require that $L_{k}$ for all three-detector combinations in the network be greater than 36 . We then set the effective SNR threshold high enough to eliminate all remaining background triggers. Figure 6 shows the quadruple coincidence rate as a function of the effective SNR, 




Figure 5. Detection efficiency of the WBCP pipeline for various sine-Gaussian simulated gravitational-wave bursts, as a function of the signal amplitude (defined by equation 18). The legend indicates the central frequency $(\mathrm{Hz})$ of the injected signal.

$\rho_{\text {eff }}$. We set a threshold on the effective SNR at 3.4. This threshold corresponds approximately to the root sum square of the matched filter SNR of 11-12 detected in the network.

To determine the detection efficiency, we then inject sine-Gaussian burst signals into the data and determine the fraction of injections detected for the selected effective SNR and likelihood thresholds. Figure 7 plots the detection efficiency as a function of the $h_{\mathrm{rss}}$ of the injected sine-Gaussians. As with the WBCP pipeline, a small fraction of the injection signals fall within periods when the data is vetoed. However, in addition to this, several injected sine-Gaussians are missed by cWB, even at the loudest injection amplitudes, because they have sky locations and polarizations where the antenna response at the Hanford detector site is very small. This means that the injection is missed by both $\mathrm{H} 1$ and $\mathrm{H} 2$. Of the two remaining detectors in the network, the noise in G1 tends to be higher than in L1. Therefore, these injected signals are only detected strongly by L1 and the trigger does not cross the selected thresholds.

\subsection{Zero-lag observations and efficiency comparison}

With the thresholds chosen using the time-shifted analysis detailed in the previous two subsections, a search for gravitational waves is performed on LIGO-GEO data between 768 and $2048 \mathrm{~Hz}$ with no time shift applied (zero-lag). No coincidences are observed above the chosen thresholds for either pipeline.

Figure 4 plots the $\Gamma$ versus $Z_{\mathrm{g}}$ scatter and $\Gamma$ distribution of the unshifted triggers from the WBCP pipeline. From figure 4(a), it is clear that there are no unshifted triggers above the predetermined thresholds of $\Gamma=4$ and $Z_{\mathrm{g}}=5$. Though the distribution of the unshifted triggers in figure $4(b)$ has an outlier at the $\Gamma=2$ histogram bin, one should bear in mind that these triggers are well below the pre-determined $\Gamma$ threshold of 4 . Using the Kolmogorov-Smirnov 


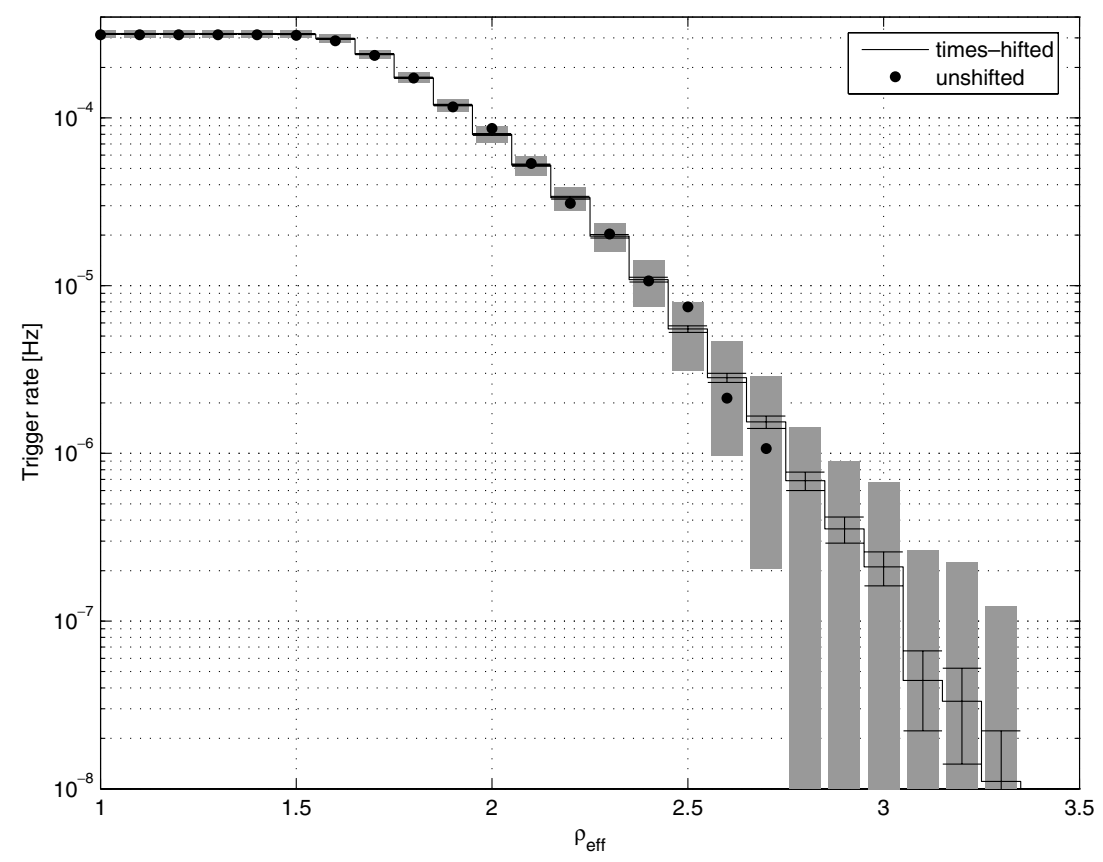

Figure 6. Rate of background triggers as a function of effective SNR for the cWB pipeline. The L1 data is shifted in 100 discrete time steps and, for each threshold value of $\rho_{\text {eff }}$, the background rate is calculated by taking an average over all 100 time shifts and plotted as the staircase plot. The $\rho_{\text {eff }}$ distribution for unshifted data is represented by black dots. As with previous figures, the error bars indicate the range corresponding to $\pm \sqrt{n} / 100$, where $n$ is the total number of triggers in each bin. Also, the grey patches indicate the standard deviation in the number of triggers at each time shift.

test, the statistical significance of the fluctuations in the $\Gamma$ distribution of the unshifted triggers is calculated to be $18 \%$, which means that the null hypothesis is accepted (assuming a standard significance threshold $5 \%$ or greater to accept the null hypothesis).

The $\rho_{\text {eff }}$ distribution of the unshifted triggers (black dots) for the cWB pipeline is shown in figure 6. The distribution of the unshifted triggers is consistent with the background distribution. No unshifted triggers were observed above the pre-determined threshold of $\rho_{\text {eff }}=3.4$. In fact, there are no unshifted triggers with $\rho_{\text {eff }}>2.7$.

With no zero-lag coincidences observed in either pipeline, we compare the sensitivities of the two pipelines. We characterize each pipeline's sensitivity by the $h_{\mathrm{rss}}^{50 \%}$ values. The $h_{\mathrm{rss}}^{50 \%}$ values for the two pipelines used on the LIGO-GEO S4 data set are given in table 2 and plotted against the strain spectral densities of the detectors in figure 8 . We note that the $h_{\mathrm{rss}}^{50 \%}$ values obtained for the cWB pipeline are 30-50\% lower than those of the WBCP pipeline. As desired, the $h_{\mathrm{rss}}^{50 \%}$ values for the cWB pipeline are also better than those for the same signals at these frequencies for a WBCP gravitational-wave burst search using only LIGO S4 data $\left(4.5 \times 10^{-21} \mathrm{~Hz}^{-1 / 2} \text { at } 849 \mathrm{~Hz} \text { and } 6.5 \times 10^{-21} \mathrm{~Hz}^{-1 / 2} \text { at } 1053 \mathrm{~Hz}\right)^{59}$ [7].

One should also bear in mind that the uncertainty in the calibration of the detector response to GW has been conservatively estimated to be $10 \%$ for LIGO and GEO 600 [23, 24]. The calibration uncertainty introduces an unknown systematic shifted in the amplitude scales in

\footnotetext{
${ }^{59}$ This search was performed in a different frequency range, 64-1600 Hz, from that reported here. Additionally, for a fairer comparison, the effects of calibration uncertainty have been removed from the values quoted here.
} 




Figure 7. Detection efficiency of the coherent Waveburst pipeline for various sine-Gaussian simulated gravitational-wave bursts, as a function of the signal amplitude (defined by equation 18). The legend indicates the central frequency $(\mathrm{Hz})$ of the injected signal.

Table 2. Table of $h_{\mathrm{rss}}^{50 \%}$ as a function of sine-Gaussian central frequencies.

\begin{tabular}{lcc}
\hline \multirow{2}{*}{$\begin{array}{l}\text { Sine-Gaussian } \\
\text { central frequency [Hz] }\end{array}$} & \multicolumn{2}{c}{$h_{\mathrm{rss}}^{50 \%}\left[\times 10^{-21} \mathrm{~Hz}^{-1 / 2}\right]$} \\
\cline { 2 - 3 } & Waveburst-CorrPower & Coherent Waveburst \\
\hline 849 & 6.8 & 3.8 \\
945 & 6.6 & 4.5 \\
1053 & 7.2 & 4.9 \\
1172 & 9.0 & 5.8 \\
1304 & 9.0 & 6.3 \\
1451 & 11.8 & 7.8 \\
1615 & 13.9 & 8.0 \\
1797 & 17.8 & 9.3 \\
2000 & 23.6 & 12.8 \\
\hline
\end{tabular}

figures 5 and 7. While the effect of calibration uncertainty is included in the gravitationalwave burst search with only LIGO S4 data, we have not included calibration uncertainty for the analysis described here. This is because, while the effect of calibration uncertainty is important for the upper limits set in [7], it is less crucial here since no upper limits have been set.

\section{Discussion}

The first joint search for gravitational-wave bursts using the LIGO and GEO 600 detectors has been presented. The search was performed using two pipelines, Waveburst-CorrPower 


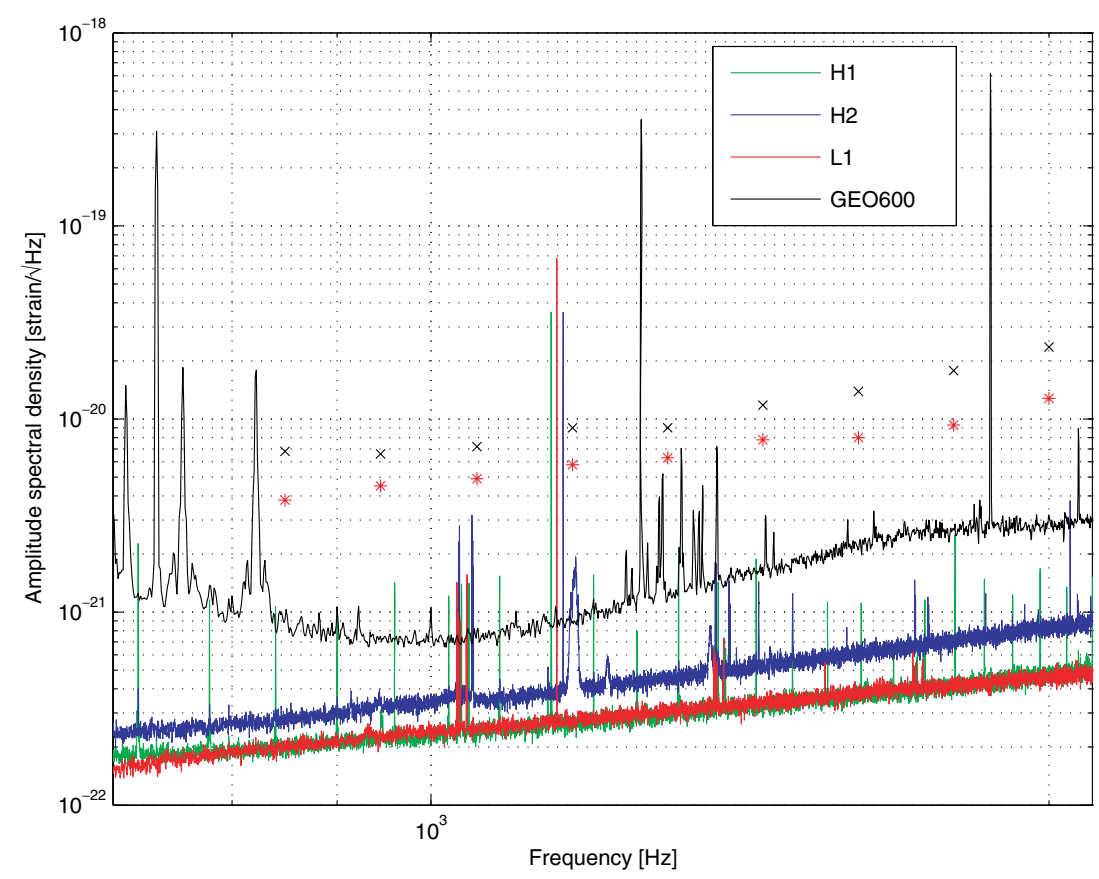

Figure 8. The $h_{\mathrm{rss}}^{50 \%}$ values for Waveburst-CorrPower (' $x$ ' markers) and coherent Waveburst ('*' markers) pipelines for sine-Gaussians of different central frequencies. Coherent Waveburst is sensitive to gravitational wave signals with amplitudes $30-50 \%$ lower than those detectable by Waveburst-CorrPower.

(WBCP) and coherent Waveburst (cWB), and targeted signals in the frequency range 768$2048 \mathrm{~Hz}$. No candidate gravitational wave signals have been identified.

The detection efficiencies of the two pipelines to sine-Gaussians have been compared. The cWB pipeline has $h_{\mathrm{rss}}^{50 \%}$ values $30-50 \%$ lower than those of the WBCP pipeline. These improved detection efficiencies are also better than those obtained for the all-sky burst search using only LIGO S4 data and the WBCP pipeline [7]. One should note, however, that the LIGO-only search was performed at a lower frequency range $(64-1600 \mathrm{~Hz})$ and optimized for the characteristics of the noise in that frequency range to maximize detection efficiency. Nonetheless, these results show that, for WBCP, the detection efficiency is limited by the least sensitive detector when applied to a network of detectors with different antenna patterns and noise levels. This is because WBCP requires that excess power be observed in coincidence by all detectors in the network. While it is certainly possible to further tune the WBCP pipeline on the LIGO-GEO S4 data to improve its sensitivity (for example, by reducing the Waveburst threshold on GEO data or not imposing quadruple coincidence [29]), we note that the cWB pipeline naturally includes detectors of different sensitivities by weighting the data with the antenna patterns and noise. Therefore, with the cWB pipeline, the detection efficiency of the network is not limited by the least sensitive detector and there is no need for pipeline tunings that are tailored for particular detector networks.

\section{Acknowledgments}

The authors gratefully acknowledge the support of the United States National Science Foundation for the construction and operation of the LIGO Laboratory and the Science 
and Technology Facilities Council of the United Kingdom, the Max-Planck-Society, and the State of Niedersachsen/Germany for support of the construction and operation of the GEO600 detector. The authors also gratefully acknowledge the support of the research by these agencies and by the Australian Research Council, the Council of Scientific and Industrial Research of India, the Istituto Nazionale di Fisica Nucleare of Italy, the Spanish Ministerio de Educación y Ciencia, the Conselleria d'Economia, Hisenda i Innovació of the Govern de les Illes Balears, the Royal Society, the Scottish Funding Council, the Scottish Universities Physics Alliance, the National Aeronautics and Space Administration, the Carnegie Trust, the Leverhulme Trust, the David and Lucile Packard Foundation, the Research Corporation, and the Alfred P Sloan Foundation. This document has been assigned LIGO Laboratory document number LIGO-P080008-B-Z.

\section{References}

[1] Sigg D (for the LIGO Scientific Collaboration) 2006 Class. Quantum Grav. 23 S51-6

[2] Lück H et al 2006 Class. Quantum Grav. 23 S71-8

[3] Acernese F et al 2006 Class. Quantum Grav. 23 S63-9

[4] Ando M and the TAMA Collaboration 2005 Class. Quantum Grav. 22 S881-9

[5] Abbott B et al 2005 Phys. Rev. D 72062001

[6] Abbott B et al 2006 Class. Quantum Grav. 23 S29-39

[7] Abbott B et al 2007 Class. Quantum Grav. 24 5343-69

[8] Abbott B et al 2005 Phys. Rev. D 72122004

[9] Klimenko S and Mitselmakher G 2004 Class. Quantum Grav. 21 S1819-30

[10] Cadonati L and Márka S 2005 Class. Quantum Grav. 22 S1159-67

[11] Cadonati L 2004 Class. Quantum Grav. 21 S1695-703

[12] Gürsel Y and Tinto M 1989 Phys. Rev. D 40 3884-939

[13] Flanagan E E and Hughes S A 1997 Phys. Rev. D 57 4566-87

[14] Rakhmanov M 2006 Class. Quantum Grav. 23 S673-85

[15] Klimenko S, Mohanty S, Rakhmanov M and Mitselmakher G 2005 Phys. Rev. D 72122002

[16] Klimenko S, Yakushin I, Mercer A and Mitselmakher G 2008 arXiv:0802.3232v1 Klimenko S, Yakushin I, Mercer A and Mitselmakher G Class. Quantum Grav. at press

[17] Wen L L and Schutz B 2005 Class. Quantum Grav. 22 S1321-35

[18] Chatterji S, Lazzarini A, Stein L, Sutton P J, Searle A and Tinto M 2006 Phys. Rev. D 74082005

[19] Mohanty S D, Rakhmanov M, Klimenko S and Mitselmakher G 2006 Class. Quantum Grav. 23 4799-809

[20] Hild S (for the LIGO Scientific Collaboration) 2006 Class. Quantum Grav. 23 S643-51

[21] Hild S, Grote H, Smith J R and Hewitson M for the GEO 600-team 2006 J. Phys.: Conf. Ser. 32 66-73

[22] Hewitson M, Grote H, Hild S, Lueck H, Ajith P, Smith J R, Strain K A, Willke B and Woan G 2005 Class. Quantum Grav. 22 4253-61

[23] Dietz A, Garofoli J, González G, Landry M, O’Reilly B and Sung M 2006 LIGO Technical Document LIGOT050262-01-D

[24] Hewitson M et al 2004 Class. Quantum Grav. 21 S1711-22

[25] Hewitson M et al 2005 Class. Quantum Grav. 22 4253-61

[26] Andersion W G, Brady P R, Creighton J D E and Flanagan E E 2001 Phys. Rev. D 63042003

[27] Daubechies I 1992 Ten lectures on wavelets (Philadelphia, PA: SIAM)

[28] Klimenko S, Yakushin I, Rakhmanov M and Mitselmakher G 2004 Class. Quantum Grav. 21 S1685-94

[29] Beauville F et al 2008 Class. Quantum Grav. 25045002 